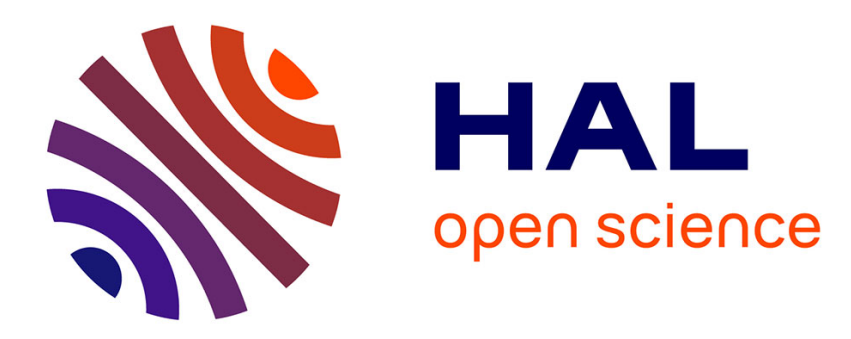

\title{
Natural Wave Control in Lattices of Linear Oscillators
} Denis Efimov, Alexander Fradkov

\section{To cite this version:}

Denis Efimov, Alexander Fradkov. Natural Wave Control in Lattices of Linear Oscillators. Systems and Control Letters, 2012, 61, pp.887-893. 10.1016/j.sysconle.2012.06.001 . hal-00704714

\section{HAL Id: hal-00704714 https://hal.inria.fr/hal-00704714}

Submitted on 6 Jun 2012

HAL is a multi-disciplinary open access archive for the deposit and dissemination of scientific research documents, whether they are published or not. The documents may come from teaching and research institutions in France or abroad, or from public or private research centers.
L'archive ouverte pluridisciplinaire HAL, est destinée au dépôt et à la diffusion de documents scientifiques de niveau recherche, publiés ou non, émanant des établissements d'enseignement et de recherche français ou étrangers, des laboratoires publics ou privés. 


\title{
Natural Wave Control in Lattices of Linear Oscillators
}

\author{
Denis V. Efimov, Alexander L. Fradkov
}

\begin{abstract}
The problem of natural wave control involves steering a lattice of oscillators towards a desired natural (i.e. zero-input) assignment of energy and phase across the lattice. This problem is formulated and solved for lattices of linear oscillators via a passivity-based approach. Numerical simulations of 1D and 2D linear lattices and a 1D lattice of nonlinear oscillators confirm the effectiveness of the proposed controls.
\end{abstract}

\section{INTRODUCTION}

Analysis and control of a complex motion in the interconnected and spatially distributed systems have been an area of intensively growing research during the last decades. It has numerous applications in many disciplines, especially in physics [6], [7], biology [28] and electrical engineering [2], [36], [39]. The problems of power system control, traffic control, control of communication networks can be solved in the framework of regulation of spatially distributed or networked systems (see special issues in the engineering journals [15]-[19]). In physics, applications of control theory for studying dynamics of complex systems lead to appearance of a new interdisciplinary area of science gradually becoming known as "Cybernetical Physics" [11].

Design and application of control strategies to manipulation of complex oscillatory and spatiotemporal patterns have become a central issue of nonlinear dynamics and physics [24] [27]. Waves propagation [1], [2], [23]-[25], [33], [37], Belousov-Zhabotinsky reaction supervision [29], [38], excitable biological tissues regulation [3], [5], [8], [40], FrenkelKontorova models [11] have been controlled by using feedback methods that points out the possibility of dynamical pattern manipulation in excitable media. Such a sort of problems arise in a variety of engineering applications ranging from the macroscopic (e.g., cross directional paper machine processes, automated highway systems, unmanned aerial vehicle or mobile robot formations, satellite constellations), to the microscopic (e.g., arrays of micro-cantilevers or nanostructures) [20]. For a spatially distributed control of a system one needs to design a stabilizing algorithm taking into account the problem of a proper control propagation over time and space. Design and analysis of an active device capable to control the mechanical waves is considered in [30], where the framework of a "mechanical wave diode" is presented. All previously mentioned works are oriented on particular control strategies synthesis and none of them studies spatially distributed systems in a systematic way. A promising approach to systematic development of control methods for complex networks or continuous distributed systems is to start with the systems of a low complexity. In this work the lattice of linear oscillators is considered as such a simple spatially distributed system.

Dynamics of many spatially distributed systems can be described by partial differential equations or, after discretization, by lattices of nonlinear oscillators [31]. Though stabilization-like control goals for lattices are well studied [14], [20], excitation of waves was considered previously only for some special cases [11], [12], [32]. In a steady state vicinity, the nonlinear lattice can be reduced to the considered lattice of linear oscillators. Such a reduction leads to many important

The first author is with the Non-A project at INRIA - LNE, Parc Scientifique de la Haute Borne, 40 avenue Halley, Bât.A Park Plaza, 59650 Villeneuve d'Ascq, France, denis.efimov@inria.fr. The second author is with CCS Lab., Institute for Problems of Mechanical Engineering, Bolshoi av., 61, V.O., St. Petersburg, 199178, Russia and St. Petersburg State University, 28 Universitetskii pr. Petrodvorets, 198504, St.Petersburg, Russia, fradkov@mail.ru. 
properties lost, for example, the breather/soliton existence phenomenon can be analyzed in nonlinear framework only [21] (that is an important area of research in physics initiated by Fermi-Pasta-Ulam (FPU) numerical experiments [6], [22]). However, as the first step for the problem formulation and a preliminary solution presentation, the lattices of linear oscillators are very useful, as we are going to show below.

In this work the problem of wave control is addressed. The existence conditions of solitons or breathers were intensively studied before, here we attempt to solve the problem of a given wave excitation starting from any initial state of a lattice. Such a control can be important for researchers working experimentally with different waves for their analysis, as well as for understanding how these waves arise in nature.

The proposed solution to the problem is based on transformation of the system to a canonic form followed by spectrum localization. The idea is to excite the desired modes in order to ensure a required oscillation amplitude and phase resetting for the prespecified vertices. Our approach to control design uses the speed-gradient method proposed in [9], [10] and extended to control of oscillations and partial stabilization in [34], [35]. The essence of the speed-gradient method is in evaluation of the speed $\dot{Q}$ of variation of the given goal function $Q$ along plant trajectories, and changing the control variables in the direction of gradient of $\dot{Q}$ with respect to the control.

The outline of the paper is as follows. The lattice equations and the problem statement are given in section 2 . The control design is performed in section 3. The results of application for 1D and 2D lattices, as well as for a 1D nonlinear FPU lattice, are presented in section 4.

\section{Problem Statement}

Consider 1D lattice of linear oscillators (the symbol $\overline{1, n}$ denotes the sequence of integers $1, \ldots, n$ ):

$$
\ddot{x}_{i}=\Omega^{2}\left(x_{i+1}+x_{i-1}-2 x_{i}\right)+b_{i} u, i=\overline{1, n}, x_{0}=x_{n+1}=0
$$

or 2D lattice

$$
\begin{gathered}
\ddot{x}_{i, j}=\Omega^{2}\left(x_{i, j+1}+x_{i, j-1}+x_{i-1, j}+x_{i+1, j}-4 x_{i, j}\right)+\mathbf{b}_{i, j}^{T} \mathbf{u}, \\
x_{i, m+1}=x_{i, 0}=x_{0, j}=x_{n+1, j}=0, i=\overline{1, n}, j=\overline{1, m},
\end{gathered}
$$

where $\Omega \in R$ is the lattices "frequency", $n>1, m>1$ define the lattices dimension, $x_{i}$ or $x_{i, j}$ correspond to 1D or 2D vertices positions, $\dot{x}_{i}, \dot{x}_{i, j}$ and $\ddot{x}_{i}, \ddot{x}_{i, j}$ stand for velocities and accelerations correspondingly, the scalar $b_{i}$ or the vector $\mathbf{b}_{i, j}$ determine appearance of the scalar control $u$ in 1D case or the vector one $\mathbf{u} \in R^{m}$ in $2 \mathrm{D}$. Typically the controls influence the lattices from one side, i.e.

$$
\begin{gathered}
b_{1} \neq 0, b_{k}=0, k=\overline{2, n} ; \\
\mathbf{b}_{1, j} \neq 0, \mathbf{b}_{k, j}=0, k=\overline{2, n}, j=\overline{1, m}
\end{gathered}
$$

in $1 \mathrm{D}$ and $2 \mathrm{D}$ cases respectively. The ND case $(\mathrm{N}>2)$ can be treated similarly.

The systems (1), (2) are linear and by enumeration of the states they can be written in the form

$$
\ddot{\mathbf{s}}=\mathbf{A s}+\mathbf{B u}, \mathbf{s} \in R^{l}
$$

for suitably defined matrices $\mathbf{A}$ and $\mathbf{B}$, where $l=n$ for a 1D lattice and $l=n m$ in 2D case (1D lattice is already in this form with $\left.\mathbf{s}=\left[x_{1}, \ldots, x_{n}\right]\right)$. The spectrum of the systems (1) or (2) is pure imaginary $\lambda_{r}= \pm \omega_{r} i, r=\overline{1, R}$ with multiplicities $p_{r}$ (the 
matrix A has eigenvalues $-\omega_{r}^{2}, r=\overline{1, R}$ ) and $\sum_{r=1}^{R} p_{r}=l$ (in $1 \mathrm{D}$ case all $p_{r}=1, r=\overline{1, R}$, but in 2D case $p_{1}=\min \{n, m\}$ ). Then there exists a linear transformation of coordinates $\mathbf{z}=\mathbf{R s}$ with nonsingular $\mathbf{R}$ composed by right eigenvectors of the matrix A (this matrix is symmetric from (1), (2) and has $l$ independent eigenvectors) such, that the systems (1), (2) can be represented in the canonical form:

$$
\begin{gathered}
\dot{z}_{k, 1}=z_{k, 2}, \dot{z}_{k, 2}=-\omega_{k}^{2} z_{k, 1}+\boldsymbol{\beta}_{k}^{T} \mathbf{u}, k=\overline{1, l}, \\
\mathbf{u} \in R^{m}, \mathcal{B}=\left[\boldsymbol{\beta}_{1} \ldots \boldsymbol{\beta}_{l}\right]^{T}=\mathbf{R}^{-1} \mathbf{B} .
\end{gathered}
$$

For each $k=\overline{1, l}$ the system (3) is called the normal mode of (1), (2). Actually the system (1), (2) has the same form as (3) with an additional coupling of neighbors and the control acting just on one layer in the lattices. In (3) all oscillators are uncoupled with the control influencing on all modes directly. The representation of lattices (3) indicates that in the uncontrolled case ( $\mathbf{u}=0$ ) the systems (1), (2) solutions are purely oscillating:

$$
\begin{gathered}
x_{i}(t)=\sum_{k=1}^{n} \alpha_{k} \sin \left(\omega_{k} t+\phi_{k}\right), i=\overline{1, n} \\
x_{k, j}(t)=\sum_{j=1}^{m} \sum_{k=1}^{n} \alpha_{k, j} \sin \left(\omega_{k, j} t+\phi_{k, j}\right), i=\overline{1, n}, j=\overline{1, m}
\end{gathered}
$$

where $\alpha_{i}, \omega_{i}, \phi_{i}$ and $\alpha_{i, j}, \omega_{i, j}, \phi_{i, j}$ are real constant parameters determined by initial conditions for 1D and 2D cases respectively. Any such solution (4) for all admissible set of parameters we will further call a natural wave for the lattices. Motivation of the term "natural" is that such waves exist in (1), (2) without an external influence. According to the lattice structure not all real values $\alpha_{i}, \phi_{i}$ and $\alpha_{i, j}, \phi_{i, j}$ for $i=\overline{1, n}, j=\overline{1, m}$ can be admissible (the frequencies $\omega_{i}, \omega_{i, j}$ are predefined by the lattice dimension and $\Omega$ ).

The problem is to create a predefined natural wave in the lattice. To this end, $m$ vertices are chosen ( $m$ is the number of the controls available) and their indices are collected in the set $\mathcal{V}, \operatorname{card}(\mathcal{V})=m($ the symbol $\operatorname{card}(\cdot)$ is stated for a set cardinality). The number $d \leq m$ of the frequencies is specified (these frequencies define the stabilized wave spectrum), their indexes are given in the set $\mathcal{F}, \operatorname{card}(\mathcal{F})=d$. The reference behavior for all vertices in $\mathcal{V}$ is defined in the form

$$
\begin{gathered}
x_{i}^{*}(t)=\sum_{k \in \mathcal{F}} A_{i, k} \sin \left(\omega_{k} t+\varphi_{i, k}\right), i \in \mathcal{V} ; \\
x_{i, j}^{*}(t)=\sum_{k \in \mathcal{F}} A_{i, j, k} \sin \left(\omega_{k} t+\varphi_{i, j, k}\right),(i, j) \in \mathcal{V}
\end{gathered}
$$

for all $t \geq 0$ in 1D and 2D cases respectively, where $A_{i, k}, \varphi_{i, k}$ and $A_{i, j, k}, \varphi_{i, j, k}$ are given constants. It is assumed that $x_{i}(t)=x_{i}^{*}(t)$ for all $i \in \mathcal{V}$ for the system (1) or $x_{i, j}(t)=x_{i, j}^{*}(t)$ for all $(i, j) \in \mathcal{V}$ for the system (2) are admissible invariant solutions with $\mathbf{u}(t) \equiv 0$ for all $t \geq 0$ (not all triples $A_{(\cdot)}, \omega_{(\cdot)}, \varphi_{(\cdot)}$ are admissible for the chosen sets $\mathcal{V}$ and $\mathcal{F}$, that follows from the lattice topology). In other words, the solutions (5) belong to the natural waves for (1), (2). From physical point of view, it is necessary to ensure the lattice oscillations of a predefined spectrum with a given profile of the wave front. Then, it is required to design a control $\mathbf{u}$ such that

$$
\begin{gathered}
\lim _{t \rightarrow+\infty}\left[x_{i}(t)-x_{i}^{*}(t)\right]=0, i \in \mathcal{V} ; \\
\lim _{t \rightarrow+\infty}\left[x_{i, j}(t)-x_{i, j}^{*}(t)\right]=0,(i, j) \in \mathcal{V} .
\end{gathered}
$$

Thus we are looking for the control that excites a predefined natural wave for the lattices (1) or (2) with the fixed behavior of 
the vertices in the set $\mathcal{V}$, i.e. a wave of a given spectrum with predefined shape. Since the wave is natural, in this case may be $\mathbf{u}(t) \rightarrow 0$ for $t \rightarrow+\infty$. It is assumed that the state of the lattice is accessible for measurements, i.e. $z_{k, 1}, z_{k, 2}$ for all $k=\overline{1, l}$.

\section{MAIN RESULT}

To solve the posed problem, a decomposition to two subproblems with two different subgoals is proposed. The first one consists in spectrum $\mathcal{F}$ localization, i.e. it is necessary to eliminate all oscillating modes in the system corresponding to the frequencies not included in $\mathcal{F}$ and to excite the desired modes $k \in \mathcal{F}$ in order to ensure the required amplitudes of oscillation $A_{(\cdot)}$ for the vertices given in $\mathcal{V}$. The second subgoal is the phase resetting for the vertices $\mathcal{V}$ (the desired phases $\varphi_{(\cdot)}$ have to be assigned), this objective deals with the wave coordination in time and space. Having the same spectrum the waves can have different forms ("standing" or "running"), the wave form can be assigned by coordination of phases for the nodes in $\mathcal{V}$. These subgoals are independent, and in general case achievement of one of them does not necessarily imply achievement of another goal. Let us consider two solutions of these subproblems utilizing the special form of (3).

\section{A. Spectrum localization}

This problem can be stated and solved for the canonical representation of the lattices (3). Each normal mode in (3) has energy or Hamiltonian function

$$
H_{k}\left(z_{k, 1}, z_{k, 2}\right)=0.5\left(z_{k, 2}^{2}+\omega_{k}^{2} z_{k, 1}^{2}\right), k=\overline{1, l}
$$

with the time derivative

$$
\dot{H}_{k}=z_{k, 2}\left(-\omega_{k}^{2} z_{k, 1}+\boldsymbol{\beta}_{k}^{T} \mathbf{u}\right)+\omega_{k}^{2} z_{k, 1} z_{k, 2}=z_{k, 2} \boldsymbol{\beta}_{k}^{T} \mathbf{u} .
$$

Then the problem of the spectrum localization can be solved by stabilization of the desired values for the energies $H_{k}^{*}$, $k=\overline{1, l}$, which we further assume given as follows: $H_{i}^{*}=0$ for all $i \notin \mathcal{F}$, and some $H_{i}^{*} \neq 0$ for all $i \in \mathcal{F}$. For brevity of presentation let $\mathbf{H}=\left[H_{1}, \ldots, H_{l}\right]^{T}, \mathbf{H}^{*}=\left[H_{1}^{*}, \ldots, H_{l}^{*}\right]^{T}$ and $S_{k}=\left\{i=\overline{1, l}: \omega_{k}=\omega_{i}\right\}, k=\overline{1, l}$ be the set of all normal modes indices with the same frequency. The proposed control design is based on speed-gradient approach [9], [10], [34], [35] with the energy-based goal function $Q(\mathbf{z})=\left[\mathbf{H}(\mathbf{z})-\mathbf{H}^{*}\right]^{T}\left[\mathbf{H}(\mathbf{z})-\mathbf{H}^{*}\right]$.

Theorem 1. For any $\mathbf{H}^{*} \in R_{+}^{l}$ consider the control

$$
\mathbf{u}=-\chi(\mathbf{y}), \mathbf{y}=\sum_{k=1}^{l}\left(H_{k}-H_{k}^{*}\right) z_{k, 2} \boldsymbol{\beta}_{k},
$$

where $\mathbf{y}^{T} \chi(\mathbf{y})>0$ for any $\mathbf{y} \in R^{m} \backslash\{0\}$ is differentiable and $\chi(0)=0, \chi^{\prime}(0) \neq 0$. Let for all $k=\overline{1, l}$ the vectors $\boldsymbol{\beta}_{s}, s \in S_{k}$ be linearly independent, then (3), (6) is partially stable with respect to the variable $\mathbf{H}(t)-\mathbf{H}^{*}$ and for all initial conditions $\mathbf{H}(0) \in \mathcal{H}=\left\{\mathbf{H} \in R_{+}^{l}: H_{i}>0, i \in \mathcal{F}\right\}$ the relation

$$
\lim _{t \rightarrow+\infty} \mathbf{H}(t)=\mathbf{H}^{*}
$$

holds, provided that the matrix $\mathbf{W}$, defined for $k=\overline{1, d}$ :

$$
W_{2 k-1,2 k}=1 ; W_{2 k-1, i}=0, i=\overline{1,2 d} \text { and } i \neq 2 k ;
$$




$$
\begin{gathered}
W_{2 k, 2 k-1}=-\omega_{k}^{2} ; W_{2 k, 2 h-1}=0, h=\overline{1, d} \text { and } h \neq k ; \\
W_{2 k, 2 h}=\chi^{\prime}(0) \boldsymbol{\beta}_{k}^{T} \boldsymbol{\beta}_{h} H_{h}^{*}, h=\overline{1, d},
\end{gathered}
$$

has all eigenvalues with positive real parts.

Proof. Consider the following Lyapunov function (proof of the theorem utilizes the passivity property of the speedgradient algorithms for conservative systems [34], [35])

$$
V(\mathbf{H})=0.5\left(\mathbf{H}-\mathbf{H}^{*}\right)^{T}\left(\mathbf{H}-\mathbf{H}^{*}\right)
$$

that for the system (3) has the time derivative $\dot{V}=\mathbf{y}^{T} \mathbf{u}$. Substitution of (6) gets $\dot{V}=-\mathbf{y}^{T} \chi(\mathbf{y}) \leq 0$, that ensures for the system (3), (6) partial stability with respect to the variable $\mathbf{H}-\mathbf{H}^{*}$ [13]. For any finite $\mathbf{H}^{*}$ this property implies boundedness of the variable $\mathbf{H}$, that in its turn guarantees the states $z_{k, 1}, z_{k, 2}, k=\overline{1, l}$ definiteness for all $t \geq 0$ and their boundedness.

Note that if $\mathbf{H}(0)=0$ then $\mathbf{u}(t)=0$ for all $t \geq 0$ and the lattices (1), (2) have no oscillations $\left(x_{i}(t)=0, x_{i, j}(t)=0\right.$ for all $t \geq 0, i=\overline{1, n}, j=\overline{1, m}$ ). To prove that in the case $\mathbf{H}(0) \neq 0$ the oscillations never die under (6) consider the system (3), (6) linearization at the origin (the origin corresponds to the case $\mathbf{H}=0$ ):

$$
\dot{\zeta}_{k, 1}=\zeta_{k, 2}, \dot{\zeta}_{k, 2}=-\omega_{k}^{2} \zeta_{k, 1}+\boldsymbol{\beta}_{k}^{T} \chi^{\prime}(0) \sum_{i \in \mathcal{F}} H_{i}^{*} \zeta_{i, 2} \boldsymbol{\beta}_{i}
$$

$k=\overline{1, l} ; \zeta_{k, 1}, \zeta_{k, 2}$ are the corresponding coordinates of the linearised system. Enumerating the states it is possible to put all modes from the set $\mathcal{F}$ as the first $2 d$ coordinates, then the matrix of the system will be low-triangular. The first block is described by the matrix $\mathbf{W}$ having $2 d$ eigenvalues with positive real parts, for $i \notin \mathcal{F}$ the blocks on the main diagonal have pure imaginary eigenvalues. Therefore, if $\mathbf{H}(0) \in \mathcal{H}$, then $H_{i}(t) \neq 0$ for all $t \geq 0$ for all $i \in \mathcal{F}$.

By construction $\dot{V}<0$ for all $\mathbf{y} \neq 0$, and $\dot{V}=0$ if and only if $\mathbf{y} \equiv 0$ and $\mathbf{u} \equiv 0$. Next, we would like to prove the system (3) (strong) observability with respect to the output $\mathbf{y}$ for the set of initial conditions $\mathbf{H}(0) \in \mathcal{H}$, i.e.

$$
\mathbf{y}(t) \equiv 0, \mathbf{u}(t) \equiv 0 \text { for all } t \geq 0 \Rightarrow \mathbf{H}(t)=\mathbf{H}^{*}, t \geq 0
$$

(owing (6) the converse trivially holds and $\mathbf{H}(t)=\mathbf{H}^{*}$ for all $t \geq 0 \Rightarrow \mathbf{y}(t) \equiv 0, \mathbf{u}(t) \equiv 0, t \geq 0$ ). If this property is satisfied, then by standard arguments $\mathbf{H}(t) \rightarrow \mathbf{H}^{*}$ with $t \rightarrow+\infty$.

Assume that $\mathbf{y}(t) \equiv 0, \mathbf{u}(t) \equiv 0$ for all $t \geq 0$. Since $\mathbf{u}(t) \equiv 0, t \geq 0$ all normal modes are isolated in (3) and for all $t \geq 0$ and for $r=\overline{1, l}$ we have

$$
H_{r}(t)=\eta_{r} ; z_{r, 1}(t)=a_{r} \sin \left(\omega_{r} t+v_{r}\right), z_{r, 2}(t)=a_{r} \omega_{r} \cos \left(\omega_{r} t+v_{r}\right), a_{r}=\sqrt{2 \eta_{r} \omega_{r}^{-2}},
$$

where $\eta_{r}$ and $v_{r}$ are the constant dependent on initial conditions. By contrary, take a set of indices $\mathcal{R}=\left\{k=\overline{1, l}: \eta_{k} \neq H_{k}^{*}\right\}$. From the output $\mathbf{y}$ definition we obtain

$$
0=\sum_{r \in \mathcal{R}}\left[\eta_{r}-H_{r}^{*}\right] a_{r} \omega_{r} \cos \left(\omega_{r} t+v_{r}\right) \boldsymbol{\beta}_{r}^{T}, t \geq 0 .
$$

It could be the case that $H_{r}^{*} \neq 0$ and $\eta_{r}=0$ for all $r \in \mathcal{R}$, then $a_{r}=0$ and the observability fails. However, from the consideration above if $H_{i}(0) \neq 0$ for all $i \in \mathcal{F}$, then it should be $\eta_{r} \neq 0$ for all $r \in \mathcal{R}$ with $H_{r}^{*} \neq 0$. Therefore for $\mathbf{H}(0) \in \mathcal{H}$ we may restrict our consideration to the case $a_{r} \neq 0, r \in \mathcal{R}$, then 


$$
\sum_{r \in \mathcal{R}} \gamma_{r} \cos \left(\omega_{r} t+v_{r}\right) \boldsymbol{\beta}_{r}^{T}=0, \gamma_{r}=a_{r} \omega_{r}\left[\eta_{r}-H_{r}^{*}\right]
$$

and the last equation can hold for all $t \geq 0$ if for all $r \in \mathcal{R}$ the equalities

$$
\sum_{s \in S_{r}} \gamma_{s} \boldsymbol{\beta}_{s}=0, v=1,2
$$

are satisfied. Since $\gamma_{s}$ are arbitrary real constants the equalities above can not be true if the vectors $\boldsymbol{\beta}_{s}, s \in S_{r}, r \in \mathcal{R}$ are linearly independent as it is claimed in the theorem conditions. We arrive at the contradiction, the set $\mathcal{R}$ is empty and the system (3) is (strongly) observable with respect to the output $\mathbf{y}$ for $\mathbf{H}(0) \in \mathcal{H}$.

Under conditions of theorem 1 the control (6) solves the problem of spectrum localization for the lattices. The conditions include the restriction on initial conditions $\mathbf{H}(0) \in \mathcal{H}$, that can be easily satisfied (if it is not the case and $\mathbf{H}(0)=0$, then a pulse $\mathbf{u}$ has to be applied exciting the lattice, next at the instant of time $t^{\prime} \geq 0$ when $\mathbf{H}\left(t^{\prime}\right) \in \mathcal{H}$ the control (6) can be activated), and the requirement on linear independence of $\boldsymbol{\beta}_{s}, s \in S_{k}, k=\overline{1, l}$. This condition is always true for 1D case, since in this case all sets $S_{k}, k=\overline{1, l}$ contain just one element (all frequencies are distinct).

\section{B. Phase resetting and wave control}

This problem can be solved for the canonical representation (3) and for the systems (1), (2) directly. In this work we will focus our attention on the case (3) only.

The desired phases $\varphi_{i, k}, i \in \mathcal{V}$ or $\varphi_{i, j, k},(i, j) \in \mathcal{V}$ correspond to the frequency $\omega_{k}, k \in \mathcal{F}$, the normal modes into the set $S_{k}$ all have the same frequency $\omega_{k}$ and different phases $v_{p}, p \in S_{k}$. The variables $x_{i}$ and $x_{i, j}$ for all $i=\overline{1, n}$, $j=\overline{1, m}$ are linear combinations of the variables $z_{k, v}, k=\overline{1, l}, v=1,2$, thus for given $H_{k}^{*}, k=\overline{1, l}$ the desired values $v_{k}^{*}$ for the phases $v_{k}, k \in \mathcal{F}$ can be derived from the phases $\varphi_{i, k}$ and $\varphi_{i, j, k}$. In this case the problem of phase resetting for $x_{i}$, $x_{i, j}$ is reduced to the problem of phase resetting for the modes $k \in \mathcal{F}$ in (3).

Each normal mode in (3) for $k=\overline{1, l}$ can be rewritten in action-angle coordinates [26]

$$
I_{k}=H_{k}\left(z_{k, 1}, z_{k, 2}\right), v_{k}=\pi / 2-\operatorname{atan}\left(\omega_{k} z_{k, 1} / z_{k, 2}\right) \Leftrightarrow z_{k, 1}=\omega_{k}^{-1} \sqrt{2 I_{k}} \cos \left(v_{k}\right), z_{k, 2}=\sqrt{2 I_{k}} \sin \left(v_{k}\right)
$$

as follows

$$
\dot{I}_{k}=\sqrt{2 I_{k}} \sin \left(v_{k}\right) \boldsymbol{\beta}_{k}^{T} \mathbf{u} ; \dot{v}_{k}=\frac{\cos \left(v_{k}\right) \boldsymbol{\beta}_{k}^{T}}{\sqrt{2 I_{k}}} \mathbf{u}-\omega_{k}, k=\overline{1, l},
$$

where $I_{k} \in R_{+}$and $v_{k} \in\left[0,2 \pi\right.$ ) (further all additions or subtractions with the phase variables $v_{k}$ are understood by modulus $2 \pi$ ). Let us stress that the action-angle representation (7) of the normal form (3) is correct for the case $\mathbf{H} \neq 0$ only. We will further accept that for the case $I_{k}=0$ always $v_{k}=0$ (formally, if $I_{k}=0$ then there is no oscillations and the phase can be defined artificially). From these equations we see that for $\mathbf{u}(t) \equiv 0$ for all $t \geq 0$ we have $v_{k}(t)=v_{k}(0)-\omega_{k} t, k=\overline{1, l}$ and the phase resetting consists in replacement of the initial conditions $v_{r}(0)$ with some predefined values $v_{r}^{*}, r \in \mathcal{F}$ which characterize the required profile of the wave in the lattice. Therefore, the objective of the phase resetting is

$$
\lim _{t \rightarrow+\infty}\left|v_{r}(t)-\left(v_{r}^{*}-\omega_{r} t\right)\right|=0, r \in \mathcal{F} \text {. }
$$


The problem of phase resetting can be easily solved for (7) applying approach similar to the presented in theorem 1, but we are more interested in simultaneous solution of the spectrum localization and phase resetting problems, i.e. we will assume that the desired values $H_{k}^{*}$ for the action variable $I_{k}, k=\overline{1, l}\left(H_{i}^{*}=0\right.$ for all $i \notin \mathcal{F}$ and $H_{i}^{*} \neq 0$ for all $\left.i \in \mathcal{F}\right)$ with the desired values $v_{r}^{*}$ for $v_{r}, r \in \mathcal{F}$ are given. Then theorem 1 admits the following extension.

Theorem 2. For any $\mathbf{H}^{*} \in R_{+}^{l}$ consider the control

$$
\begin{gathered}
\mathbf{u}=-\chi(\boldsymbol{\psi}), \\
\boldsymbol{\psi}=\sum_{k=1}^{l}\left(I_{k}-H_{k}^{*}\right) \sqrt{2 I_{k}} \sin \left(v_{k}\right) \boldsymbol{\beta}_{k}+\sum_{r \in \mathcal{F}}\left(v_{r}-v_{r}^{*}+\omega_{r} t\right) \cos \left(v_{r}\right) \boldsymbol{\beta}_{r} / \sqrt{2 I_{r}},
\end{gathered}
$$

where $\boldsymbol{\psi}^{T} \chi(\boldsymbol{\psi})>0$ for any $\boldsymbol{\psi} \in R^{m} \backslash\{0\}, \chi(0)=0, \sup _{\boldsymbol{\psi} \in R^{m}}|\chi(\boldsymbol{\psi})|<+\infty$. Let for all $k=\overline{1, l}$ the vectors $\boldsymbol{\beta}_{s}$, se $S_{k}$ be linearly independent, then the system (7), (8) is partially stable with respect to the variables $\mathbf{H}(t)-\mathbf{H}^{*}, v_{r}(t)-v_{r}^{*}+\omega_{r} t$ $r \in \mathcal{F}$ and for all initial conditions the relations hold:

$$
\lim _{t \rightarrow+\infty} \mathbf{H}(t)=\mathbf{H}^{*}, \lim _{t \rightarrow+\infty}\left[v_{r}(t)-v_{r}^{*}+\omega_{r} t\right]=0, r \in \mathcal{F}
$$

Proof. The control (8) is always finite and even for the case $\mathbf{H}=0$ the solutions of the system (7), (8) are well defined at least locally. Consider the following Lyapunov function

$$
V=0.5\left[\sum_{k=1}^{l}\left(I_{k}-H_{k}^{*}\right)^{2}+\sum_{r \in \mathcal{F}}\left(v_{r}-v_{r}^{*}+\omega_{r} t\right)^{2}\right]
$$

in this case the function $V$ is explicitly time-varying and its time derivative has form $\dot{V}=\boldsymbol{\psi}^{T} \mathbf{u}$. Substitution of (8) gives $\dot{V}=-\boldsymbol{\psi}^{T} \chi(\boldsymbol{\psi}) \leq 0$, that ensures the system (7), (8) partial stability with respect to the variables $\mathbf{H}-\mathbf{H}^{*}$ and $v_{r}(t)-v_{r}^{*}+\omega_{r} t, r \in \mathcal{F}$ [13]. Boundedness of $\mathbf{H}$ ensures that the system (7) state trajectories are well defined and bounded for all $t \geq 0$.

By construction $\dot{V}<0$ for all $\psi \neq 0$, and $\dot{V}=0$ if and only if $\psi \equiv 0$ and $\mathbf{u} \equiv 0$ (since the control (8) is time-varying, even $\mathbf{H}=0$ doest not imply $\mathbf{u}=0$ ). Next, the last thing to prove is the system (7) (strong) observability with respect to the output $\boldsymbol{\psi}$, i.e.

$$
\begin{gathered}
\boldsymbol{\psi}(t) \equiv 0, \mathbf{u}(t) \equiv 0 \text { for all } t \geq 0 \Rightarrow I_{k}(t)=H_{k}^{*}, k=\overline{1, l}, \\
v_{r}(t)=v_{r}^{*}-\omega_{r} t, r \in \mathcal{F} \text { for all } t \geq 0
\end{gathered}
$$

(the converse is obviously satisfied). If the observability is true, then the desired attractivity is substantiated.

Let $\psi(t) \equiv 0, \mathbf{u}(t) \equiv 0$ for all $t \geq 0$. Since $\mathbf{u}(t) \equiv 0, t \geq 0$ all systems in (7) are isolated and for all $t \geq 0$ and $r=\overline{1, l}$ the relations $I_{r}(t)=\eta_{r}, v_{r}=\kappa_{r}-\omega_{r} t$ hold, where $\eta_{r}$ and $\kappa_{r}$ are the constants dependent on initial conditions. By contrary, assume that there exist some sets of indices $\mathcal{R}=\left\{k=\overline{1, l}: \eta_{k} \neq H_{k}^{*}\right\}$ and $\mathcal{P}=\left\{r \in \mathcal{F}: \kappa_{r} \neq v_{r}^{*}\right\}$, then from the output $\boldsymbol{\psi}$ definition we for all $t \geq 0$ obtain

$$
\sum_{k \in \mathcal{R}} \gamma_{k} \sin \left(\kappa_{k}-\omega_{k} t\right) \boldsymbol{\beta}_{k}+\sum_{r \in \mathcal{P}} \alpha_{r} \cos \left(\kappa_{r}-\omega_{r} t\right) \boldsymbol{\beta}_{r}=0, \gamma_{k}=\left(\eta_{k}-H_{k}^{*}\right) \sqrt{2 \eta_{k}}, \alpha_{r}=\left(\kappa_{r}-v_{r}^{*}\right) / \sqrt{2 \eta_{r}}, t \geq 0 .
$$

The last equation can hold for all $t \geq 0$ if for all $r \in \mathcal{R} \cup \mathcal{P}$ the equalities $\sum_{s \in S_{r}} \gamma_{s} \boldsymbol{\beta}_{s}=0, \sum_{s \in S_{r}} \alpha_{s} \boldsymbol{\beta}_{s}=0$ are satisfied. Since $\gamma_{s}, \alpha_{s}$ are arbitrary real constants the equalities above can not be true if the vectors $\boldsymbol{\beta}_{s}, s \in S_{r}, r \in \mathcal{R} \cup \mathcal{P}$ are 
linearly independent as it is claimed in the theorem conditions. We arrive at the contradiction, the sets $\mathcal{R}, \mathcal{P}$ are empty and the system (7) is (strongly) observable with respect to the output $\boldsymbol{\psi}$.

Under conditions of theorem 2 the control (8) solves the problems of spectrum localization and phase resetting for the lattices (1), (2). Therefore, a natural wave stabilization with desired parameters is ensured. The conditions include the requirement on linear independence of $\boldsymbol{\beta}_{s}, s \in S_{k}, k=\overline{1, l}$ only. Since the control (8) is time-varying the problem of oscillation death is naturally solved: at the point $\mathbf{H}=0$ the bounded control (8) automatically generates an exciting pulse. Again the vectors $\boldsymbol{\beta}_{s}$ are always linearly independent in 1D case.

\section{EXAMPLES}

a.

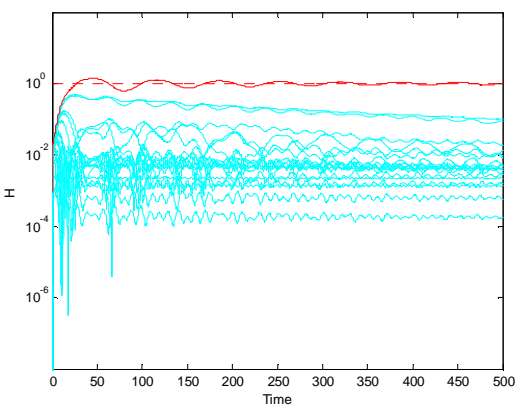

b.

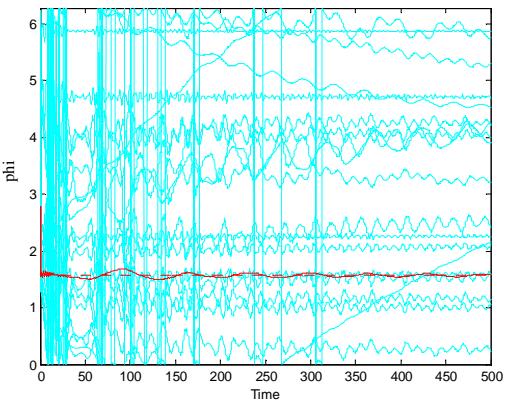

c.

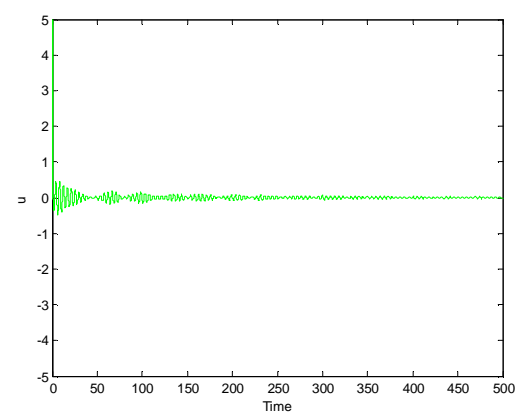

Fig. 1. Results of simulation for $1 \mathrm{D}$ case

\section{A. 1D lattice}

The results of the control (8) application for 1D lattice with $n=20$ is presented in Fig. 1 with $\chi(y)=5 \tanh (y), b_{1}=1$, $b_{k}=0, k=\overline{2, n}$ and $\Omega=1$. One mode with the frequency $\omega=2.14$ is chosen into the set $\mathcal{F}$, the desired value of the phase is $\pi / 2$. In Fig 1 , a the energies $H_{r}(t), r=\overline{1, l}$ are plotted, in Fig. 1,b the variables $\varphi_{r}(t)=v_{r}(t)+\omega_{r} t, r=\overline{1, l}$ are presented (these variables have to converge to constant values, in particular $\varphi_{r}(t) \rightarrow v_{r}^{*}+\omega_{r} t$ for $r \in \mathcal{F}$ ) and the control is 
shown in Fig. 1,c. The red solid curves correspond to regulated variables $H_{r}, \varphi_{r}$, the red dash lines represent the corresponding desired values $H_{r}^{*}, v_{r}^{*}$.

\section{B. 2 D lattice}

The results of the control (8) application for $2 \mathrm{D}$ lattice with $n=m=10$ is presented in Fig. 2 with $\chi(y)=5$ tanh $(y)$. The vectors $\mathbf{b}_{1, j}, j=\overline{1, m}$ form the identity matrix (each node in the input layer is regulated by its own control) and $\mathbf{b}_{k, j}=0, k=\overline{2, n}, j=\overline{1, m}$ and $\Omega=1$. The frequency $\omega_{1}=2$ has maximal multiplicity $p_{1}=n=10$ in this case, and all normal modes with this frequency are chosen to reach the level of energy equals 1 (the corresponding desired phases are uniformly distributed from 0 and $\pi$ ). In Fig 2,a the energies $H_{r}(t), r=\overline{1, l}$ are presented, in Fig. 2,b the variables $\varphi_{r}(t)=v_{r}(t)+\omega_{r} t, r=\overline{1, l}$ are shown (these variables have to converge to constant values, in particular $\varphi_{r}(t) \rightarrow v_{r}^{*}+\omega_{r} t$ for $r \in \mathcal{F}$ ) and the controls are plotted in Fig. 2,c. The red solid curves correspond to regulated variables $H_{r}, \varphi_{r}$, the red dash lines represent the corresponding desired values $H_{r}^{*}, v_{r}^{*}$.

a.

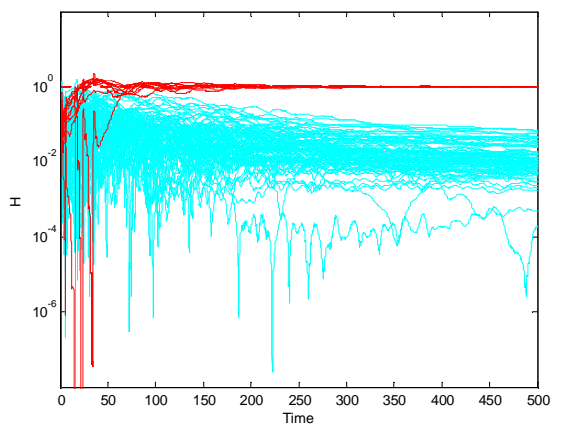

b.
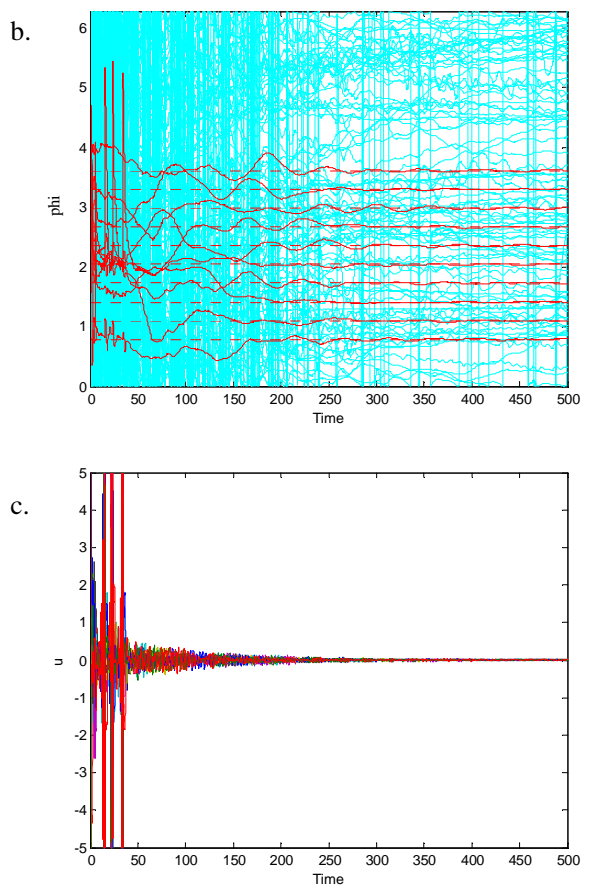

Fig. 2. Results of simulation for $2 \mathrm{D}$ case 


\section{FPU nonlinear lattice}

Finally consider a nonlinear 1D lattice from the FPU experiment [4], [22]:

$$
\ddot{x}_{i}=\Omega^{2}\left(x_{i+1}+x_{i-1}-2 x_{i}\right)+\alpha\left[\left(x_{i+1}-x_{i}\right)^{2}-\left(x_{i-1}-x_{i}\right)^{2}\right]+b_{i} u, i=\overline{1, n}, x_{0}=x_{n+1}=0
$$

where $\alpha>0$ is a parameter, and all symbols have the same meaning as previously. The difference between (1) and (9) consists in appearance of the nonlinear coupling proportional to $\alpha$. The system (9) admits soliton or breather solutions.

Let us apply to (9) the controls developed for the system (1) to demonstrate that the linear approximation of lattice oscillations is rather reliable. To this purpose, we chose the same parameters of the system (9) as for (1) in the first simulation: $n=20 ; \chi(y)=5 \tanh (y) ; b_{1}=1, b_{k}=0, k=\overline{2, n} ; \Omega=1$ and $\alpha=0.333$. The mode with the frequency $\omega=2.14$ is chosen into the set $\mathcal{F}$, the desired value of the corresponding phase is $\pi / 2$. The results of the lattice (9) simulation are shown in Fig. 3. In Fig 3 , a the energies $H_{r}(t), r=\overline{1, l}$ are plotted, in Fig. 3,b the variables $\varphi_{r}(t)=v_{r}(t)+\omega_{r} t, r=\overline{1, l}$ are presented and the control is shown in Fig. 3,c. The red solid curves correspond to regulated variables $H_{r}, \varphi_{r}$, the red dash lines represent the corresponding desired values $H_{r}^{*}, v_{r}^{*}$.

a.

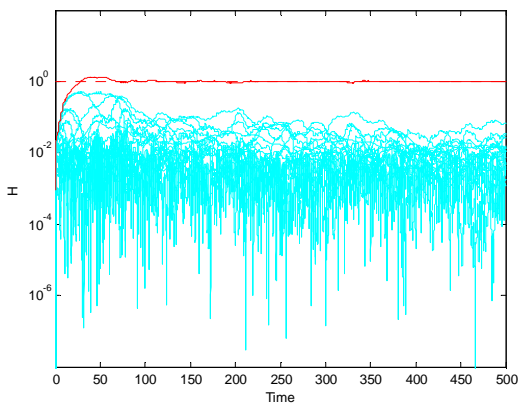

b.

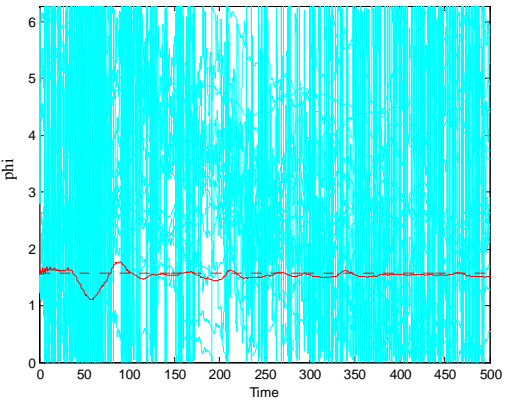

c.

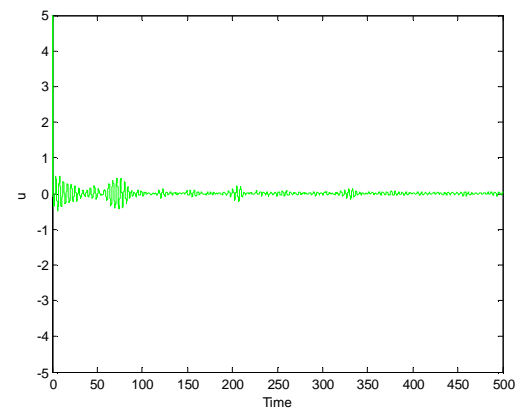

Fig. 3. Results of simulation for the FPU case 
Comparing the results presented in figures 1 and 3 note that the convergence of $H_{r}$ in the FPU case is less monotone but still takes place, while the phases $\varphi_{r}$ do not approach a constant values except the one from the set $\mathcal{F}$ (for the nonlinear case the phase definition and its dynamics are more complicated, formally the frequency of oscillations in (9) depends of the system current energy). Nevertheless, the control converges to zero asymptotically. These results demonstrate that the proposed wave regulation approach is rather robust, and it is relevant for weakly nonlinear lattices also (even if the deviations from the steady state have significant amplitudes).

\section{CONCLUSION}

The problem of natural waves stabilization at the desired energy levels for lattices of linear oscillators is posed and solved. Passivity-based approach is used to derive the control algorithms. Analytical applicability conditions of the method are established. Simulation results confirm efficiency of the proposed methodology.

Interestingly to note, that it is hard to solve the posed problem for the original linear systems (1) or (2). However, applying the nonlinear transformation we obtain a straightforward solution for the nonlinear representation (7). The proposed control law is also nonlinear. The phase resetting problem is addressed.

Some preliminary results of application of the proposed method to a nonlinear FPU lattice are reported. A theoretical extension of the proposed approach to nonlinear case is rest for a future work.

\section{REFERENCES}

[1] Alonso S., Sagués F., Mikhailov A.S. Taming Winfree Turbulence of Scroll Waves in Excitable Media. Science, 299(5613), 2003, pp. $1722-1725$.

[2] Chow S.N., Mallet-Paret J. Pattern formation and spatial chaos in lattice dynamical systems. I, II, IEEE Trans. Circuits Systems, 42, 1995, pp. 746756.

[3] Dahlem M., Mair T., Müller S.C. Spatio-temporal Aspects of a Dynamical Disease: Waves of Spreading Depression. In: Function and regulation of cellular systems. Birkhauser, Basel, 2003.

[4] Dauxois T., Peyrard M., Ruffo S. The Fermi-Pasta-Ulam "numerical experiment": history and pedagogical perspectives. European Journal of Physics, 26(5), 2005, S3-S11.

[5] Davidenko J.M., Pertsov A.V., Salomonsz R., Baxter W., Jalife J. Stationary and drifting spiral waves of excitation in isolated cardiac muscle. Nature, 355, 1992, pp. 349-51.

[6] English L.Q., Palmero F., Sievers A.J., et al. Traveling and stationary intrinsic localized modes and their spatial control in electrical lattices. Physical Rev. E, 81(4), 2010, № 046605 .

[7] Feurer T., Vaughan J.C., Nelson K.A. Spatiotemporal coherent control of lattice vibrational waves. Science, 299(5605), 2003 , pp. $374-377$.

[8] Falcke M., Li Y., Lechleiter J.D., Camacho P. Modeling the dependence of the period of intracellular Ca2+ waves on SERCA expression. Biophys J., 85(3), 2003, pp. 1474-1481.

[9] Fradkov A.L. Speed-gradient scheme and its application in adaptive control problems. Automation and Remote Control, 40(9), 1979, pp. 1333-1342.

[10] Fradkov A. L. Integro-differentiating velocity gradient algorithms. Sov. Physics-Doklady, 31(2), 1986, pp. 97-98.

[11] Fradkov A.L. Cybernetical Physics: From Control of Chaos to Quantum Control. Springer, 2007.

[12] Fradkov A.L., Andrievsky B. Control of Wave Motion in the Chain of Pendulums. Proc. 17th IFAC World Congress, Seoul, 2008 , pp. $3136-3141$.

[13] Fradkov A.L., Miroshnik I.V., Nikiforov V.O. Nonlinear and adaptive control of complex systems. Kluwer Academic Publishers, 1999.

[14] Gadaleta S., Dangelmayr G. Control of 1-D and 2-D coupled map lattices through reinforcement learning. Proc. 2nd Intern. Conf. Control of Oscillations and Chaos, 2000. pp.109-112.

[15] Special issue on Control of Complex Networks". IEEE Control Syst.Mag., 21(6),December 2001.

[16] Special issue on Control of Complex Networks". IEEE Control Syst. Mag., 22(1), February 2002.

[17] Special Section "Complex Networked Control Systems". IEEE Control Syst. Mag., August 2007.

[18] Special issue on Technology of Networked Control Systems. Proc. IEEE, 95(1), January 2007. 
[19] Special issue on Networked Control Systems. IEEE Trans. Automat. Contr., September 2004.

[20] Jovanovic M.R., Bamieh B. Lyapunov-based distributed control of systems on lattices. IEEE Trans. Automat. Contr., 50(4), 2005 , pp. $422-433$.

[21] Karsten A., Pikovsky A. Traveling waves and compactons in phase oscillator lattices. Chaos, 18(3), 2008,, p. 037118.

[22] Kevrekidis P.G. Nonlinear Waves in Lattices: Past, Present, Future. IMA Journal of Applied Mathematics, 76(1970), 2010 , p. 35.

[23] Kheowan O.-U., Kantrasiri S., Wilairat P. Spiral wave dynamics under feedback control derived from a variety of sensory domains Physical Review E, 70, 2004.

[24] Kheowan O.-U., Kantrasiri S., Wilairat P., Storb U., Müller S.C. Spiral wave dynamics under feedback control derived from a variety of sensory domains. Physical Review E, 70, 2004, p. 046221.

[25] Kheowan O.-U., Zykov V., Müller S. Transition from local to global feedback control of spiral wave dynamics. Phys. Chem. Chem. Phys., 4, 2002, pp. $1334-1338$.

[26] Landau L.D., Lifshitz E.M. Mechanics, 3rd. ed., Pergamon Press, 1976.

[27] Lebiedz D., Brandt-Pollmann U. Manipulation of self-aggregation patterns and waves in a reaction-diffusion system by optimal boundary control strategies. Physical Review Letters, 91(20), 2003, p. 208301.

[28] Levskaya A., Weiner O.D., Lim W.A., et al. Spatiotemporal control of cell signalling using a light-switchable protein interaction. Nature, 461(7266), 2009, pp. 997-1001.

[29] Müller S.C., Plesser T., Hess B. The Structure of the Core of the Spiral Wave in the Belousov-Zhabotinskii Reaction. Science, 230, 1985, pp. 661-663.

[30] Nauclér P., Lundberg B., Söderström T. A mechanical wave diode: Using feedforward control for one-way transmission of elastic extensional waves. IEEE Trans. Contr. Syst. Technol., 15(4), 2007, pp. 715-724.

[31] Pikovsky A., Rosenblum M., Kurths J. Synchronization: A Universal Concept in Nonlinear Sciences. Cambridge: Cambridge University Press, 2001.

[32] Polushin I.G. Energy control of the Toda lattice. Proc. 2nd Intern. Conf. Control of Oscillations and Chaos, 2000. pp. 26-28.

[33] Sakurai T., E. Mihaliuk, F. Chirila, Showalter K. Design and Control of Wave Propagation Patterns in Excitable Media. Science, 296, 2002, pp. 2009-2012.

[34] Shiriaev A.S., Fradkov A.L. Stabilization of invariant sets for nonlinear non-affine systems. Automatica, 36(11), 2000, pp. 1709-1715.

[35] Shiriaev A.S., Fradkov A.L. Stabilization of invariant sets for nonlinear systems with applications to control of oscillations. Intern. J. Robust Nonlinear Control, 11, 2001, pp. 215-240.

[36] Steur E., Nijmeijer H. Synchronization in Networks of Diffusively Time-Delay Coupled (Semi-)Passive Systems. IEEE Trans. Circuits and Systems I, 58(6), 2011, pp. 1358-1371.

[37] Zykov V.S., Bordiougov G., Brandtstädter H., Gerdes I., Engel H. Global control of spiral wave dynamics in an excitable domain of circular and elliptical shape. Phys. Rev. Lett., 92, 2004, p. 018304.

[38] Winfree A.T. The Geometry of Biological Time. Springer Verlag: Berlin, 1980.

[39] Wu W., Zhou W.J., Chen T.P. Cluster Synchronization of Linearly Coupled Complex Networks Under Pinning Control. IEEE Trans. Circuits and Systems I, 56(4), 2009, pp. 829-839.

[40] Zhang H., Patel N. Spiral wave breakdown in an excitable medium model of cardiac tissue. Chaos, Solitons \& Fractals, 5(3-4), 1995, pp. 635-639. 